\title{
Case of invasive nontypable Haemophilus influenzae respiratory tract infection with a large quantity of neutrophil extracellular traps in sputum
}

\author{
This article was published in the following Dove Press journal: \\ Journal of Inflammation Research \\ 17 December 2012 \\ Number of times this article has been viewed
}

\author{
Shigeto Hamaguchi ${ }^{1, *}$ \\ Masafumi Seki',* \\ Norihisa Yamamoto' \\ Tomoya Hirose ${ }^{2}$ \\ Naoya Matsumoto 2 \\ Taro Irisawa ${ }^{2}$ \\ Ryosuke Takegawa ${ }^{2}$ \\ Takeshi Shimazu ${ }^{2}$ \\ Kazunori Tomono' \\ 'Division of Infection Control \\ and Prevention, ${ }^{2}$ Department of \\ Traumatology and Acute Critical \\ Medicine, Osaka University Graduate \\ School of Medicine, Osaka, Japan \\ *These authors contributed equally \\ to this work
}

\begin{abstract}
Haemophilus influenzae type b was once the most common cause of invasive $H$. influenzae infection, but the incidence of this disease has decreased markedly with introduction of conjugate vaccines to prevent the disease. In contrast, the incidence of invasive infection caused by nontypable $H$. influenzae has increased in the US and in European countries. Neutrophil extracellular traps (NETs) are fibrous structures released extracellularly from activated neutrophils during inflammation, including in pneumonia, and rapidly trap and kill pathogens as a first line of immunological defense. However, their function and pathological role have not been fully investigated. Here, we report a case of fatal nontypable $H$. influenzae infection with severe pneumonia and bacteremia in an adult found to have a vast amount of NETs in his sputum. The patient had a two-day history of common cold-like symptoms and was taken to the emergency room as a cardiopulmonary arrest. He recovered temporarily, but died soon afterwards, although appropriate antibiotic therapy and general management had been instituted. Massive lobular pneumonia and sepsis due to nontypable $H$. influenzae was found, in spite of $H$. influenzae type b vaccine being available. His sputum showed numerous bacteria phagocytosed by neutrophils, and immunohistological staining indicated a number of NETs containing DNA, histone H3, and neutrophil elastase. This case highlights an association between formation of NETs and severe respiratory and septic infection. An increase in severe nontypable $H$. influenzae disease can be expected as a result of "pathogen shift" due to increased use of the H. influenzae type b vaccine in Japan.
\end{abstract}

Keywords: neutrophil extracellular traps, sepsis, pneumonia, Haemophilus influenzae, type b, nontypable

\section{Introduction}

Haemophilus influenzae is classified as serotypes a-f and nontypable strains based on the presence of different or undetectable capsular polysaccharides. ${ }^{1} \mathrm{H}$. influenzae type $\mathrm{b}$ is the most common cause of invasive $H$. influenzae infection. Nontypable $H$. influenzae is usually found to be the causative organism in mild mucosal infections, such as bronchopneumonia or otitis media, but has rarely been identified as the causative pathogen in invasive fatal infection. As the use of $H$. influenzae type b conjugate vaccines has widened, the incidence of invasive $H$. influenzae type b disease has decreased markedly. In contrast, the incidence of invasive infection caused by nontypable $H$. influenzae has increased, and this phenomenon has become known as "pathogen shift".,2,3
Correspondence: Masafumi Seki Division of Infection Control and Prevention, Osaka University Hospital, 2-15 Yamadaoka, Suita City, Osaka, 565-087I, Japan

Tel +81668795093

Fax +8I6 68795094

Email seki@hp-infect.med.osaka-u.ac.jp 
Neutrophil extracellular traps (NETs) are fibrous structures that have recently been reported as one of the important immunological components released extracellularly from activated neutrophils to trap and kill pathogens. ${ }^{4,5}$ However, the significance of NETs in vivo in association with the clinical pathology of infection is still not fully understood.

Here we report a patient who had severe $H$. influenzae infection and a large amount of NETs in his sputum, suggesting a relationship between NETs and the pathogenesis of severe respiratory inflammation.

\section{Case report}

A 76-year-old man with liver cirrhosis caused by chronic hepatitis $\mathrm{C}$ was admitted to the intensive care unit. He had a history of thoracoplasty for the treatment of pulmonary tuberculosis. Two days before admission, he experienced common cold-like symptoms and consulted a doctor at a nearby medical clinic. However, his symptoms worsened, and on the morning of admission he lost consciousness at home. He was transported immediately to our hospital by ambulance, during which he required cardiopulmonary resuscitation. A return of circulation was observed on arrival at our hospital. His vital signs included a consciousness level of E1V1M1 on the Glasgow Coma Scale, body temperature $34.4^{\circ} \mathrm{C}$, blood pressure $63 / 46 \mathrm{mmHg}$, and heart rate 66 beats per minute. Loss of light reflex was observed. Laboratory studies on admission revealed signs of inflammation (white blood cell count 13,330 cells/mL, C-reactive protein $2.16 \mathrm{mg} / \mathrm{dL}$ ), elevated liver enzyme levels (aspartate transaminase $459 \mathrm{U} / \mathrm{L}$, alanine transaminase $171 \mathrm{U} / \mathrm{L}$ ), and mild renal dysfunction (creatinine $1.38 \mathrm{ng} / \mathrm{dL}$ ). A chest radiograph revealed right upper and lower lobe consolidation with collapse of the left chest wall caused by his previous thoracoplasty (Figure 1).

Gram staining of endotracheal-aspirated sputum revealed a large number of neutrophils, and light microscopy at high magnification $(1000 \times)$ showed numerous fibrous constituents (Figure 2A-E). The same specimens were analyzed using immunohistochemistry with DAPI (4',6-diamidino2-phenylindole) staining (Invitrogen, Carlsbad, CA) for DNA detection (Figure 2A), anti-human histone $\mathrm{H} 3$ mouse monoclonal antibody (MABI0001, MAB Institute Inc, Sapporo, Japan) for histone (Figure 2B), and anti-human neutrophil elastase rabbit polyclonal antibody (GTX72042, GeneTex Inc, Irvine, CA) for neutrophil elastase (Figure 2C). Figure $2 \mathrm{D}$ is a merged photograph of Figure $2 \mathrm{~A}-\mathrm{C}$. The specimens were visualized using a fluorescence microscope (BZ-9000, Keyence Corporation, Osaka, Japan).

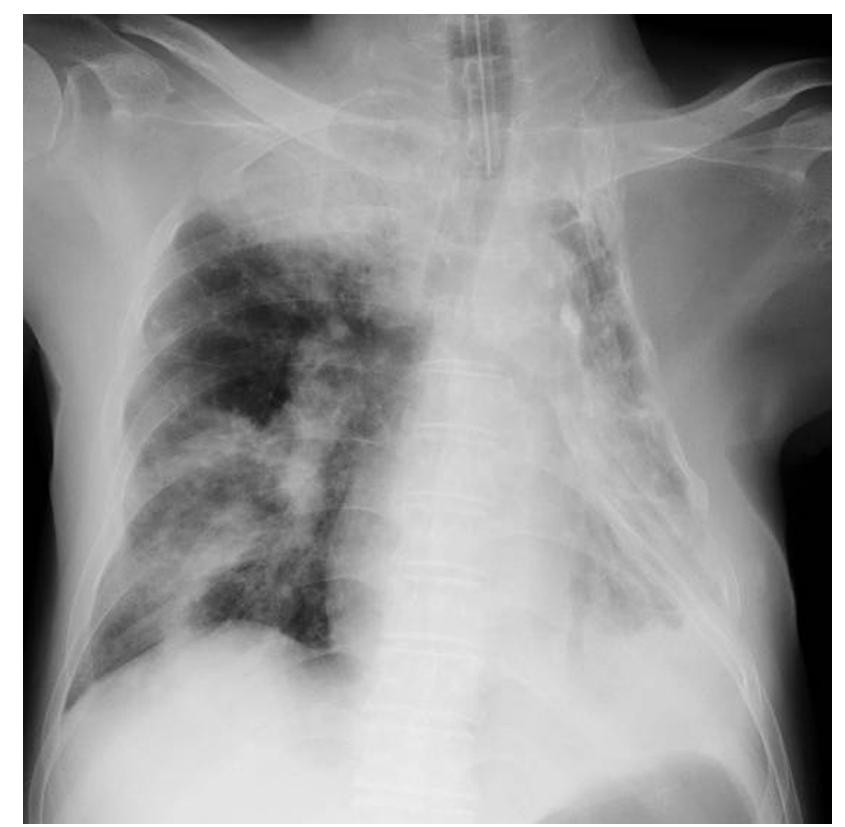

Figure I Chest radiograph on admission.

Notes: Large consolidations and multiple lobular shadows were seen in the upper and middle fields of the right lung. The left lung was operated and collapsed due to old tuberculosis.

The findings suggested that the fibrous structures in sputum were mainly NETs, defined as extracellular stringlike structures that are simultaneously immunoreactive for DNA, histone H3, and neutrophil elastase. ${ }^{6}$ A number of Gram-negative coccobacilli, some of which had been phagocytosed by neutrophils, were observed at high magnification (1000×, Figure 3).

Despite immediate administration of broad-spectrum antibiotics upon admission, the patient died on the day following hospitalization. H. influenzae was later isolated and detected in both sputum and blood from the culture performed on admission. The blood culture results identified nontypable H. influenzae (Denka Seiken Co, Ltd, Tokyo, Japan), and we diagnosed invasive disease caused by nontypable $H$. influenzae infection with multilobar pneumonia and bacteremia/sepsis in a patient with liver cirrhosis post thoracoplasty.

\section{Discussion}

This patient had very severe pneumonia (Figure 1) with bacteremia as a result of $H$. influenzae infection, and died soon after admission, in spite of appropriate antibiotic therapy and general management. Gram-staining of an endotrachealaspirated sputum specimen showed a large number of fibrous structures and many neutrophils. Within the same specimen, immunostaining visualized the major components of NETs, ie, DNA, histone $\mathrm{H} 3$, and neutrophil elastase, enabling us 

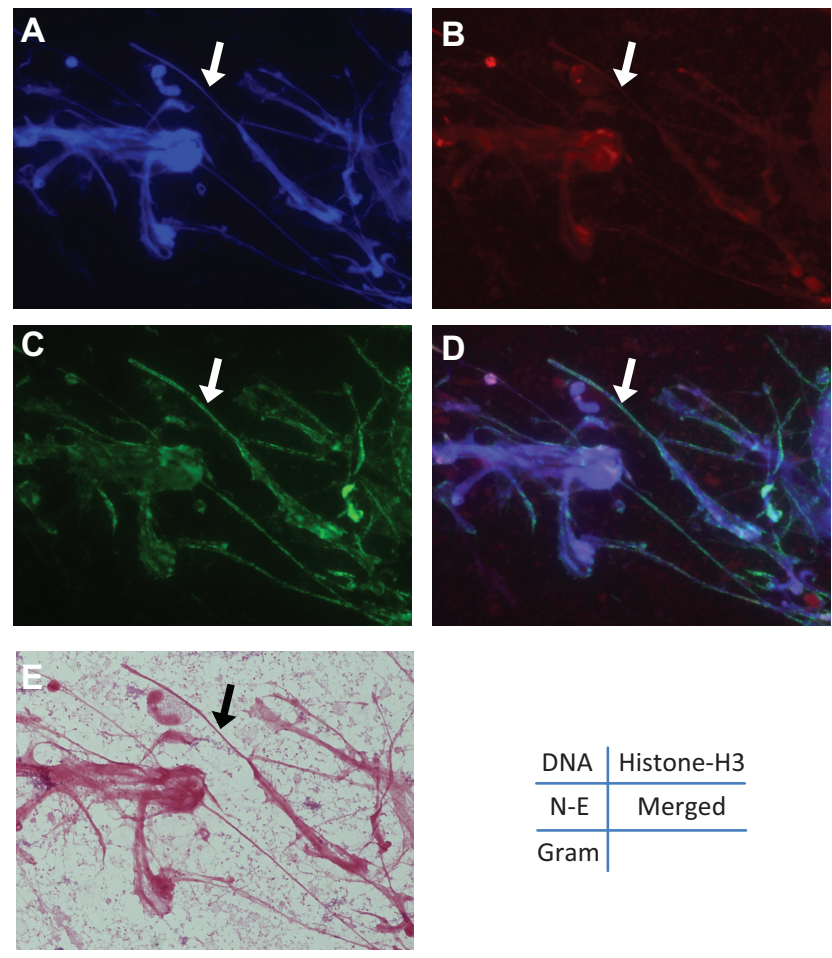

\begin{tabular}{c|c} 
DNA & Histone-H3 \\
\hline N-E & Merged \\
\hline Gram &
\end{tabular}

Figure 2 Formation of NETs in the sputum of this patient. (A) 4',6-diamidino-2 phenylindole staining at high magnification $(1000 \times)$. (B) Immunohistochemistry against anti-human histone-H3 mouse monoclonal antibody. (C) Immunohistochemistry against anti-human neutrophil elastase rabbit polyclonal antibody. (D) Merged photograph of $(\mathbf{A}-\mathbf{C})$. These pictures indicate that the fibrous constituents are NETs. (E) Gram-staining of endotracheal-aspirated sputum at low magnification (I000x). Note: Arrow indicates numerous fibrous constituents.

Abbreviation: NETs, neutrophil extracellular traps.

to confirm that these fibrous structures comprised mainly NETs (Figure 2). NET formation has been only recently recognized as a function of neutrophils. ${ }^{4}$ It was shown that activated neutrophils release DNA fibers decorated with antimicrobial proteins, which form NETs that bind, disarm, and kill pathogens extracellularly.

Bianchi et al have reported that NET formation and nicotinamide adenine dinucleotide phosphate (NADPH) oxidase contributed to fungal infection in a patient with chronic granulomatous disease and impaired phagocyte NADPH oxidase function. ${ }^{7}$ They also used gene therapy in this patient to restore production of NETS and the ability of neutrophils to eliminate Aspergillus nidulans conidia and hyphae, leading to a rapid cure of invasive pulmonary aspergillosis. ${ }^{8}$ Furthermore, Yost et al found impaired NET formation may be a critical facet of a common developmental immunodeficiency that predisposes newborn infants to infection. ${ }^{9}$

We recently reported that purulent sputum from patients with acute respiratory infection contains abundant long NETs, and that the amount of NETs decreases gradually as the clinical symptoms and inflammation subside. ${ }^{6}$ Samples from our

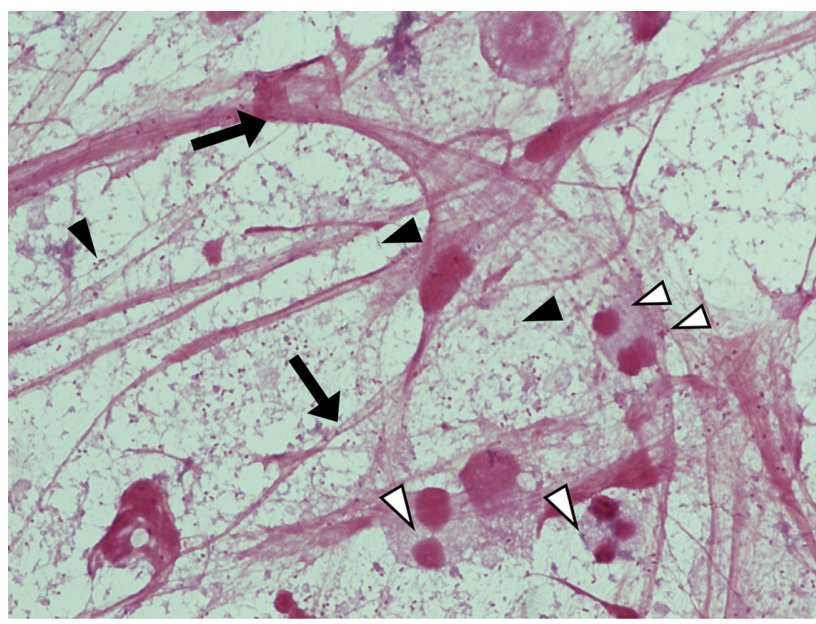

Figure 3 Gram-staining of endotracheal-aspirated sputum.

Notes: A large number of Gram-negative coccobacilli (black arrowheads), including a proportion phagocytosed by neutrophils (white arrowheads) and numerous fibrous NETs (arrows) are observed at higher magnification (1000X).

Abbreviation: NETs, neutrophil extracellular traps.

patient showed many NETs in his sputum, and this might be a characteristic finding of lower respiratory infection caused by invasive nontypable $H$. influenzae. We quantified the NET length by computer software (Neurolucida ${ }^{\circledR}, \mathrm{MBF}$ Bioscience, Williston, VT), and found a correlation between the lengths of these fibrous structures and the number of inflammatory cytokines in the serum of patients with acute respiratory infection (paper in preparation). Compared with these cases, this nontypable $H$. influenzae cases showed very severe chest $\mathrm{x}$-ray findings and a lot of bacteria and structures including NETs in sputum.

The escape mechanism from NETs may be one of the virulent factors of invasive infections. Walker et al reported that group A streptococcus, which facilitates its escape from NETs via a quorum of virulent genes (including mutated covRS) with corporative roles, is an example of natural selection exerted by the innate immune system generates hypervirulent bacterial variants with increased risk of systemic dissemination. ${ }^{10}$

In addition, there has been a report showing that nontypable $H$. influenzae can induce formation of NETs, but that NETs may not be a significant determinant of clearance of nontypable $H$. influenzae during chronic and recurrent otitis media. ${ }^{11}$ Rather, colonies of nontypable invasive $H$. influenzae with biofilm phenotypes appear to survive within the NET structure, and seem to promote rather than inhibit bacterial persistence. Inappropriate NET formation may also contribute to inflammatory tissue damage, and potentially function as a "double-edged sword". The relationship between NETs and thrombus formation in tissue damage, including after trauma, have been also reported. ${ }^{12-14}$ 
Nontypable $H$. influenzae progressing to invasive disease might possess some unique virulent factor to escape NETs. The relationship between pathogens and NETs in respiratory specimens is worth further study. We have not had any other lethal nontypable $H$. influenzae cases in adults, but we have encountered lethal pneumonia due to $H$. influenzae type $\mathrm{d}$ cases (data not shown). These cases and other nonlethal cases did not show any microbiological differences, including in colony formation. We plan to analyze the genomic and biochemical differences between lethal and nonlethal H. influenzae isolates.

Our patient was an immunocompromised host with liver cirrhosis, and therefore at high risk of infection. Reasons for frequent occurrence of infection in this disease include decreased reticuloendothelial function, which may due to a decrease in the numbers or functioning of Kupffer cells, reduced IgM antibodies and complement levels, decreased numbers and functioning of neutrophils, and reduced synthesis of opsonization factors. ${ }^{15}$ These factors are potentially exacerbated by portosystemic shunting due to increased portal venous pressure and impaired lymph flow. Furthermore, this patient had not received $H$. influenzae type b vaccination because this vaccine has only been available for children since 2007 in Japan.

In conclusion, we have reported here a case of fatal nontypable $H$. influenzae infection including severe pneumonia and sepsis with a vast amount of NET structures in sputum. Further investigation of the virulence of this pathogen and immune reactions including NET formation, is needed.

\section{Disclosure}

The authors report no conflicts of interest in this work.

\section{References}

1. Kroll JS, Booy R. Haemophilus influenzae: capsule vaccine and capsulation genetics. Mol Med Today. 1996;2:160-165.

2. Kastrin T, Paragi M, Kolman J, et al. Characterisation of invasive Haemophilus influenzae isolates in Slovenia, 1993-2008. Eur J Clin Microbiol Infect Dis. 2010;29:661-668.

3. Resman F, Ristovski M, Ahl J, et al. Invasive disease caused by Haemophilus influenzae in Sweden 1997-2009: evidence of increasing incidence and clinical burden of non-type b strains. Clin Microbiol Infect. 2011;17:1638-1645.

4. Brinkmann V, Reichard U, Goosmann C, et al. Neutrophil extracellular traps kill bacteria. Science. 2004;303:1532-1535.

5. Logters T, Margraf S, Altrichter J, et al. The clinical value of neutrophil extracellular traps. Med Microbiol Immunol. 2009;198:211-219.

6. Hirose T, Hamaguchi S, Matsumoto N, et al. Dynamic changes in the expression of neutrophil extracellular traps in acute respiratory infections. Am J Respir Crit Care Med. 2012;185:1130-1131.

7. Bianchi M, Niemiec MJ, Siler U, Urban CF, Reichenbach J. Restoration of anti-Aspergillus defense by neutrophil extracellular traps in human chronic granulomatous disease after gene therapy is calprotectindependent. J Allergy Clin Immunol. 2011;127:1243-1252.

8. Bianchi M, Hakkim A, Brinkmann V, et al. Restoration of NET formation by gene therapy in CGD controls aspergillosis. Blood. 2009;114:2619-2622.

9. Yost CC, Cody MJ, Harris ES, et al. Impaired neutrophil extracellular trap (NET) formation: a novel innate immune deficiency of human neonates. Blood. 2009;113:6419-6427.

10. Walker MJ, Hollands A, Sanderson-Smith ML, et al. DNase Sda1 provides selection pressure for a switch to invasive group A streptococcal infection. Nat Med. 2007;13:981-985.

11. Juneau RA, Pang B, Weimer KED, et al. Nontypeable Haemophilus influenzae initiates formation of neutrophil extracellular traps. Infect Immune. 2011;79:431-438.

12. Kambas K, Mitroulis I, Apostolidou E, et al. Autophagy mediates the delivery of thrombogenic tissue factor to neutrophil extracellular traps in human sepsis. PLoS One. 2012;7:e45427.

13. Meng W, Paunel-Görgülü A, Flohé S, et al. Deoxyribonuclease is a potential counter regulator of aberrant neutrophil extracellular traps formation after major trauma. Mediators Inflamm. 2012;2012:149560.

14. Fuchs TA, Brill A, Duerschmied D, et al. Extracellular DNA traps promote thrombosis. Proc Natl Acad Sci U S A. 2010;107:15880-15885.

15. Wyke RJ. Problems of bacterial infection in patients with liver disease. Gut. 1987;28:623-641.
Journal of Inflammation Research

\section{Publish your work in this journal}

The Journal of Inflammation Research is an international, peer-reviewed open-access journal that welcomes laboratory and clinical findings on the molecular basis, cell biology and pharmacology of inflammation including original research, reviews, symposium reports, hypothesis formation and commentaries on: acute/chronic inflammation; mediators of inflamma-

\section{Dovepress}

tion; cellular processes; molecular mechanisms; pharmacology and novel anti-inflammatory drugs; clinical conditions involving inflammation. The manuscript management system is completely online and includes a very quick and fair peer-review system. Visit http://www.dovepress.com/ testimonials.php to read real quotes from published authors. 